\title{
An Essential, Hyperconnected, Local Geometric Morphism that is not Locally Connected
}

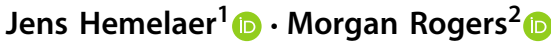

Received: 26 October 2020 / Accepted: 15 December 2020 / Published online: 15 February 2021

(c) The Author(s) 2021

\section{Abstract}

Thomas Streicher asked on the category theory mailing list whether every essential, hyperconnected, local geometric morphism is automatically locally connected. We show that this is not the case, by providing a counterexample.

Keywords Grothendieck topos · Essential · Hyperconnected · Local · Locally connected · Geometric morphism

We show that an essential, hyperconnected, local geometric morphism is not necessarily locally connected, by constructing a counterexample. This answers a question asked by Thomas Streicher on the category theory mailing list. Our counterexample arises from our earlier work [1] and work-in-progress regarding properties of geometric morphisms $\operatorname{PSh}(M) \rightarrow \operatorname{PSh}(N)$ for monoids $M$ and $N$.

We thank Thomas Streicher for the interesting question and the subsequent discussion regarding this counterexample. Further, we would like to thank the anonymous reviewer for their suggestions, especially for the different proof that $f$ is not locally connected (which replaces our previous, longer proof).

The first named author is a postdoctoral fellow of the Research Foundation - Flanders (File number $1276521 \mathrm{~N}$ ). The second named author was supported in this work by INdAM and the Marie Sklodowska-Curie Actions as a part of the INdAM Doctoral Programme in Mathematics and/or Applications Cofunded by Marie Sklodowska-Curie Actions.

Communicated by Thomas Streicher.

$凶$ Morgan Rogers

mrogers@uninsubria.it

Jens Hemelaer

jens.hemelaer@uantwerpen.be

1 Department of Mathematics, University of Antwerp, Middelheimlaan 1, 2020 Antwerp, Belgium

2 Istituto Nazionale di Alta Matematica, Università degli Studi dell’Insubria, Via Valleggio n. 11, 22100 Como, CO, Italy 


\section{The counterexample}

Let $M$ be the monoid with presentation $\left\langle e, x: e^{2}=e, x e=x\right\rangle$. Note that each element of $M$ can be written as either $x^{n}$ or $e x^{n}$ for some $n \in\{0,1,2, \ldots\}$. Further, let $N$ be the free monoid on one variable $a$, so $N=\left\{1, a, a^{2}, \ldots\right\}$. Consider the monoid morphism $\phi: M \rightarrow N$ which on generators is given by $\phi(e)=1$ and $\phi(x)=a$. If we interpret $M$ and $N$ as categories, then $\phi$ is a functor. There is an induced essential geometric morphism

$$
\operatorname{PSh}(M) \stackrel{f}{\longrightarrow} \operatorname{PSh}(N)
$$

given by functors

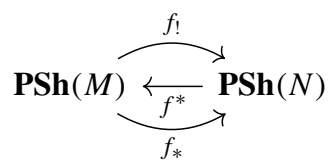

with the following description, for $X$ in $\mathbf{P S h}(M)$ and $Y$ in $\mathbf{P S h}(N)$ :

- $f_{!}(X) \simeq X \otimes_{M} N$ where $N$ has left $M$-action defined by $m \cdot n=\phi(m) n$ for $m \in M$ and $n \in N$, and right $N$-action defined by multiplication;

- $f^{*}(Y) \simeq Y$ with right $M$-action defined as $y \cdot m=y \cdot \phi(m)$ for $y \in Y$ and $m \in M$;

- $f_{*}(X) \simeq \mathcal{H o m}_{M}(N, X)$, where $N$ has right $M$-action given by $n \cdot m=n \phi(m)$ for $n \in N$ and $m \in M$, and $\mathcal{H o m}_{M}(N, X)$ is the set of morphisms of right $M$-sets $g: N \rightarrow Y$; the right $N$-action on $\mathcal{H o m}_{M}(N, X)$ is defined as $(g \cdot n)\left(n^{\prime}\right)=g\left(n n^{\prime}\right)$ for $g \in \mathcal{H o m}_{M}(N, X)$ and $n, n^{\prime} \in N$.

For definitions and background regarding tensor products and Hom-sets, in the context of sets with a monoid action, we refer to [1, Subsection 1.2].

Proposition 1 The geometric morphism $f$ is hyperconnected and local.

Proof Because $\phi$ is surjective, it follows that $f$ is hyperconnected, see [2, Example A.4.6.9]. We now show that $f$ is local. Because $f$ is connected (even hyperconnected), it follows from [3, Corollary 3.3] that $f$ is local if and only if $f_{*}$ has a further right adjoint $f^{!}$. Note that there is an isomorphism of right $M$-sets $N \cong e M$, so $f_{*}(X) \simeq \mathcal{H o m}_{M}(e M, X)$. A map of right $M$-sets $e M \rightarrow X$ is completely determined by the image of $e$, and this image can be any element of $X e$. So we see that $f_{*}(X) \simeq X e$, with the right $N$-action defined as $b \cdot a=b \cdot x$ for $b \in X e$. In other words, $f_{*}(X) \simeq X \otimes_{M} M e$, where $M e$ has a left $M$-action by multiplication and a right $N$-action given by $m \cdot a=m \cdot x$ for $m \in M e$. From the tensor-hom adjunction (see e.g. [1, Proposition 1.5]), we now know that $f_{*}$ has a right adjoint given by $f^{!}(Y) \simeq \mathcal{H o m}_{N}(M e, Y)$ for $Y$ in $\operatorname{PSh}(N)$. It follows that $f$ is local.

Remark 2 As suggested by the reviewer, we can give an explicit description of the functor $f^{!}$as follows. We define $f^{!}(Y) \simeq Y \times Y$, with the right $M$-action defined as

$$
(s, t) \cdot x=(t, t \cdot a), \quad(s, t) \cdot e=(s, s \cdot a) .
$$

for $(s, t) \in Y \times Y$. To see that this agrees with the description $f^{!}(Y) \simeq \mathcal{H o m}_{N}(M e, Y)$ as above, note that $M e \cong N \sqcup N$ as right $N$-set. The right $M$-action on

$$
f^{!}(Y) \simeq \mathcal{H o m}_{N}(M e, Y)
$$

is the one induced by the left $M$-action on $M e$ (by multiplication). 
If $f$ were locally connected, then in particular $f^{*}$ would preserve exponential objects. We will show that this is not the case, and as a result $f$ is not locally connected.

\section{Proposition 3 The functor $f^{*}$ does not preserve exponential objects.}

Proof Let $2=1 \sqcup 1$. We claim that the comparison map

$$
f^{*}\left(2^{N}\right) \longrightarrow f^{*}(2)^{f^{*}(N)}
$$

is not an isomorphism. In our case, the comparison map is the map

$$
\begin{aligned}
& \mathcal{H o m}_{N}(N \times N, 2) \longrightarrow \mathcal{H o m}_{M}(M \times N, 2) \\
& \gamma \mapsto \gamma \circ g
\end{aligned}
$$

where $g(m, n)=(\phi(m), n)$. We can identify $\operatorname{Hom}_{N}(N \times N, 2)$ with the set of complemented sub- $N$-sets of $N \times N$, and similarly we can identify $\operatorname{Hom}_{M}(M \times N, 2)$ with the set of complemented sub-M-sets of $M \times N$. The comparison map then sends $S \subseteq N \times N$ to $g^{-1}(S) \subseteq M \times N$. Now consider the (right) sub- $M$-set

$$
T=\left\{\left(e x^{n+1}, a^{n}\right): n \geq 0\right\} \subseteq M \times N .
$$

To verify that this is a complemented subset, note that there is a degree function

$$
\begin{aligned}
& \operatorname{deg}: M \times N \rightarrow \mathbb{Z} \\
& \operatorname{deg}\left(x^{i}, a^{j}\right)=\operatorname{deg}\left(e x^{i}, a^{j}\right)=i-j
\end{aligned}
$$

such that $\operatorname{deg}(y \cdot m)=\operatorname{deg}(y)$ for all $y \in M \times N, m \in M$. Further, if $y \in M \times N$ is of the form $y=\left(x^{n+1}, a^{n}\right)$, then both $y \cdot x=\left(x^{n+2}, a^{n+1}\right)$ and $y \cdot e=y$ are again of this form. So $T$ is complemented, but it is not of the form $g^{-1}(S)$ for some $S \subseteq N \times N$, because it contains the element $(e x, 1)$ but not the element $(x, 1)$. So the comparison map is not surjective, and as a result $f^{*}$ does not preserve exponential objects.

Corollary 4 The geometric morphism $f$ is not locally connected.

Funding Open Access funding provided by Università degli Studi dell'Insubria

Data availability statement Not applicable.

Open Access This article is licensed under a Creative Commons Attribution 4.0 International License, which permits use, sharing, adaptation, distribution and reproduction in any medium or format, as long as you give appropriate credit to the original author(s) and the source, provide a link to the Creative Commons licence, and indicate if changes were made. The images or other third party material in this article are included in the article's Creative Commons licence, unless indicated otherwise in a credit line to the material. If material is not included in the article's Creative Commons licence and your intended use is not permitted by statutory regulation or exceeds the permitted use, you will need to obtain permission directly from the copyright holder. To view a copy of this licence, visit http://creativecommons.org/licenses/by/4.0/.

\section{References}

1. Hemelaer, J., Rogers, M.: Monoid properties as invariants of toposes of monoid actions. Appl. Categ. Struct. (2020). https://doi.org/10.1007/s10485-020-09620-y

2. Johnstone, P.T.: Sketches of an Elephant: A Topos Theory Compendium. The Clarendon Press, Oxford University Press, Oxford (2002)

3. Johnstone, P.T.: Remarks on punctual local connectedness. Theory Appl. Categ. 25(3), 51-63 (2011) 
Publisher's Note Springer Nature remains neutral with regard to jurisdictional claims in published maps and institutional affiliations. 